Editorial

\title{
Tendencias en Tecnologías de Información y Comunicación
}

\section{Trends in Information and Communication Technologies}

\author{
Jezreel Mejiaํㅜ, Mirna Muñoz ${ }^{1}$, \\ \{jmejia, mirna.munoz \}@cimat.mx \\ ${ }^{1}$ Centro de Investigación en Matemáticas- Unidad zacatecas, Av. Universidad No. 222, 98068, \\ Zacatecas, México
}

DOI: 10.17013/risti.21.ix-xii

\section{Introducción}

Los artículos presentados en este número fueron seleccionados de los artículos aceptados en el Quinto Congreso Internacional de Mejora de Procesos Software (CIMPS) 2016, realizado en la Ciudad de Aguascalientes, Aguascalientes, México del 12 al 14 de octubre del 2016. Este congreso ha tenido una taza de aceptación del 30\% de los artículos enviados.

En este número de la Revista Ibérica de Sistemas y Tecnologías de la Información (RISTI), aborda temas relacionados a "Tendencias en Tecnologías de Información y Comunicación". Los artículos presentan versiones extendidas y mejoradas de las versiones originales que han sido presentados en el congreso CIMPS 2016.

Tendencias en Tecnologías de Información y Comunicación

El uso de las Tecnologías de Información y Comunicación (TICs) se han vuelto una estrategia competitiva que permite a las organizaciones posicionarse dentro de su mercado de actuación. Además, la evolución, avance y uso de las TICs dentro de cualquier tipo de organización ha creado nuevos dominios de interés:

Dentro del ciclo de desarrollo de software hay dos partes fundamentales que son las pruebas y estimación para el desarrollo de software:

- En las pruebas del software que son importantes en el ciclo de desarrollo han surgido modelos como TPI, TMMI, entre otros para ser abordadas. Un estándar 
para tratar de estandarizar el proceso de pruebas es el estándar ISO/IEC 29119. La ISO/IEC/IEEE 29119 es el nuevo estándar internacional para guiar el proceso de pruebas de software (ISO/IEC, 2013) y está constituido actualmente por (ISO/ IEC, 2013): 4 publicaciones (Conceptos y definiciones, Procesos, Documentación y Técnicas) y otras 3 en desarrollo (Pruebas dirigidas por palabras clave, Modelo de evaluación de procesos, y Revisión de productos de trabajo).

- La estimación es una métrica principal para el desarrollo y seguimiento del mismo proyecto. Un método que ha venido siendo utilizado son los puntos de caso de uso para la estimación de esfuerzo en el desarrollo de un proyecto de software. Este consiste en la estimación del tiempo de desarrollo mediante la asignación de "pesos" o valores que se puedan contabilizar en el tiempo total estimado para el desarrollo del proyecto de software en específico (Recalde \& Lopez, 2010).

En el contexto de técnicas de gamificación han sido utilizadas más allá del dominio de juegos tradicionales, esto trae una gran oportunidad hacia otras áreas como lo es la Ingeniería del Software (IS). En términos generales, se refiere a la aplicación de elementos de juego en contexto que no son de juego mejorando la experiencia y compromiso de los participantes (Korn, Funk, \& Schmidt, 2015). Es por ello, que en IS está aplicándose, ya que el desarrollo de software el trabajo es realizado en equipos.

Con respecto a la explotación de la información que generan las organizaciones para la toma de decisiones están siendo utilizadas la minera de procesos, el análisis de clúster y los sistemas de recuperación de información:

- La minera de procesos a través de un conjunto de datos conformado por un registro de eventos tiene como finalidad el descubrimiento de procesos (Van der Aalst, 2016). Por lo tanto, la minera de procesos puede ser utilizado para la exploración de los procesos históricos dentro de una organización para la implementación de mejoras.

- Otro aspecto importante en la minería, pero específicamente en datos es el análisis de clúster que tiene por objetivo identificar y descubrir datos de tal manera que elementos asignados a un mismo grupo sean similares entre sí, mientras que elementos pertenecientes a grupos distintos sean disímiles (Yue, 2016).

- Los sistemas de recuperación de información ofrecen mecanismos que permitan a las empresas adquirir, producir y transmitir, al menor costo, datos e información con los atributos de calidad, precisión y validez, que sean útiles para la toma de decisiones (Arévalo, 2007; Mejia et al., 2014). Los sistemas de recuperación de información tratan con texto, generalmente escrito en lenguaje natural, no bien estructurado y semánticamente ambiguo (Lara Navarra \& Martínez Usero, 2006). En este contexto los sistemas expertos están siendo utilizados por las empresas para complementar el uso de información para la toma de decisiones y predecir preferencias y predicciones de comportamiento como en la minería o preferencia en consumidores (Yang et al., 2016; Shabankareh \& Hezarkhani, 2016).

Finalmente, en relación al crecimiento y uso de internet en nuestros días, es muy común que los servicios electrónicos y aplicaciones web soliciten información a través de un registro web para solicitar información como dirección, edad, nombre, genero, entre otros datos principales. Por consecuencia, los usuarios comparten información sensible 
al momento de enviar su información. El problema con este registro es que, los usuarios de Internet revelan información (Joinson, Buchanan, \& Paine Schofield, 2010). Esta información puede ser utilizada para fines delictivos o comerciales.

Los artículos publicados en este número de RISTI tratan de cubrir las Tendencias en Tecnologías de Información y Comunicación que actualmente están siendo abordadas.

\section{Estructura}

En el primer artículo se presenta un análisis exploratorio sobre la adopción de prácticas de pruebas de software de la ISO/IEC 29119-2. Para ello, los autores realizaron un análisis usando un estudio de caso múltiple de cuatro unidades de análisis, considerando dos tipos de organizaciones: Organizaciones que actuaron como fábricas de software en un esquema contractual y Organizaciones que formaron parte de un equipo de desarrollo de software.

El segundo articulo tiene como objetivo determinar los factores que afectan la precisión de la estimación del esfuerzo en proyectos de software, usando Puntos de Caso de Uso como método de estimación. Por lo que, analizan un total de 37 estudios primarios como resultado de una revisión de la literatura.

El tercer articulo presenta los resultados de una revisión sistemática, enfocada en el uso de gamificación para crear un equipo, reduciendo el tiempo de integración y por lo tanto mejorar su rendimiento.

El cuarto articulo muestra la aplicación y utilidad de la minería de procesos en la ingeniería de software, mostrando una serie de casos de estudio que ilustran aplicaciones sobre el proceso y el producto. El principal resultado de este artículo es que el uso de minería de procesos facilita la evaluación y auditoría de procesos de software.

El quinto articulo propone el diseño e implementación de un esquema de visualización para modelos de clústeres, en el contexto de un proceso de minería de datos. Por lo que, los autores proponen un esquema de visualización llamado VIMC, y se basa en cuatro características: visualización interactiva, combinación de técnicas de minería de datos, artefactos gráficos ad-hoc, y uso de métricas

En el sexto articulo los autores presentan una solución a la administración de documentos digitales de ADD Intelligence in Aviation. Para ello, se desarrolló el sistema Be Intelligent que utiliza un índice invertido para indexar y recuperar fácilmente los contenidos de documentos que satisfacen una consulta hecha en lenguaje natural.

El séptimo articulo describe un método para crear explicaciones basadas en las conclusiones parciales por las que transita un Sistema Experto, para concluir a cerca del comportamiento de un sistema dinámico complejo. Las entradas a este método por los autores son: el árbol de inferencia y el conocimiento del dominio representado mediante una Ontología.

Finalmente, en el octavo artículo se presenta la exposición de problemas de privacidad y seguridad existentes en el proceso de registro en sitios web como las redes sociales, describiendo los problemas que puede ocasionar la falta de seguridad y privacidad en estos sitios. 


\section{Agradecimientos}

Agradecemos al director de la Revista Álvaro Rocha por otorgar la oportunidad para dar a conocer los trabajos más relevantes presentados en el congreso CIMPS 2016. Asimismo, expresamos nuestra gratitud a los autores de los trabajos publicados, lo cual hizo posible este número en RISTI. Damos la enhorabuena a todos aquellos autores cuyos artículos han sido publicados en este número de RISTI.

\section{Referencias}

Arévalo, J. A. (2007). Gestión de la Información, Gestión de Contenidos y Conocimiento. In II Jornadas de Trabajo del Grupo SIOU (pp. 1-15). Universidad de Salmanaca.

ISO/IEC. (2013). ISO/IEC/IEEE 29119-1:2013 Software and systems engineering - Software testing - Part 1: Concepts and definitions. Geneva, Switzerland: International Organization for Standardization.

ISO/IEC. (2013). ISO/IEC/IEEE 29119-2:2013 Software and systems engineering -- Software testing -- Part 2: Test processes. Geneva, Switzerland: International Organization for Standardization.

Joinson, A. R.-d., Buchanan, T., \& Paine Schofield, C. (2010). Privacy, Trust, and SelfDisclosure Online. Human-Computer Interaction, 1-24.

Korn, O., Funk, M., \& Schmidt, A. (2015). Towards a Gamification of Industrial Production. A Comparative Study in Sheltered Work Environments, (JUNE), 84-93.

Lara Navarra, P., \& Martínez Usero, J. A. (2006). Agentes Inteligentes en la Búsqueda y Recuperación de Información (Segunda Ed). Barcelona, España: Planeta-UOC, S. L.

Mejia, J., Muñoz, M., \& Rocha, Á. (2014). La Mejora de Procesos de Software a través de la Gestión de Procesos, el Outsourcing y la Adopción de Nuevas Tecnologías de Información y Comunicación. RISTI - Revista Ibérica de Sistemas e Tecnologias de Informação, E1, vii-xi.

Recalde, O. M., \& Lopez, A. M. (2010). Estimación Basada en Casos de Uso UCP-Use Case Points (Doctoral dissertation, Tesis de Maestría. Universidad Politécnica de Madrid, Madrid).

Shabankareh, M., \& Hezarkhani, A. (2016). Copper potential mapping in Kerman copper bearing belt by using ANFIS method and the input evidential layer analysis. Arabian Journal of Geosciences, 9(5), 1-12

Van der Aalst, W. M. (2016). Process mining: data science in action. Springer.

Yang, Y., Fu, C., Chen, Y. W., Xu, D. L., \& Yang, S. L. (2016). A belief rule based expert system for predicting consumer preference in new product development. Knowledge-Based Systems, 94, 105-113.

Yue, W. (2016). Research on the Clustering Analysis Algorithm for Data Mining. RISTI Revista Ibérica de Sistemas e Tecnologias de Informação, E6, 209-221. 